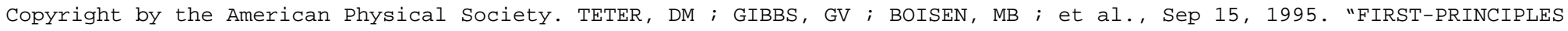

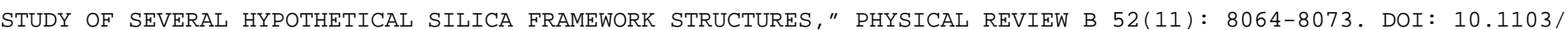
PhysRevB.52.8064.

\title{
First-principles study of several hypothetical silica framework structures
}

\author{
D.M. Teter \\ Department of Materials Science and Engineering, Virginia Tech, Blacksburg, Virginia 24061 \\ and Geophysical Laboratory, Carnegie Institution of Washington, Washington, D.C. 20015-1305 \\ G.V. Gibbs \\ Department of Materials Science and Engineering, Department of Geological Sciences, \\ Virginia Tech, Blacksburg, Virginia 24061 \\ M.B. Boisen Jr. \\ Department of Mathematics, Virginia Tech, Blacksburg, Virginia 24061 \\ D.C. Allan \\ Fundamental Research, Corning Incorporated, Corning, New York 14831 \\ and Department of Physics, The Ohio State University, Columbus, Ohio 43210 \\ M.P. Teter \\ Fundamental Research, Corning Incorporated, Corning, New York 14831 \\ and Laboratory of Atomic and Solid State Physics, Cornell University, Ithaca, New York 14853-2501
}

(Received 29 March 1995)

\begin{abstract}
Several hypothetical silica structures have been generated using a simulated-annealing strategy with an $a b$ initio based covalent-bonding potential. First-principles total-energy pseudopotential methods have been used to examine several promising hypothetical structures and to compare their structural parameters, cohesive energies, and bulk moduli with those of low quartz, low cristobalite, silica sodalite, and stishovite. The cohesive energies of these hypothetical structure types are found to be equivalent to those of low quartz, low cristobalite, and silica sodalite, and significantly lower than that of stishovite.
\end{abstract}

\section{INTRODUCTION}

One of the most challenging computational problems in materials science is the first-principles derivation of the stable and metastable structure types that can crystallize in a given chemical system and the prediction of their properties. ${ }^{1,2}$ In particular, a derivation of the stable and metastable structure types for the silica system and a prediction of their properties is of interest, because of the use of silica in technology and its abundance in nature. Not only is silica widespread in nature, being found principally as the stable structure type quartz and, to a much lesser extent as cristobalite, tridymite, coesite, and stishovite, but it also adopts a large variety of metastable structure types, some of which occur in nature and others which have been synthesized in the laboratory. Besides its industrial uses such as filters, insulators, and microstrain control devices, silica has also been put to good use in computational physics as a relatively simple but challenging system for testing computer models and modeling strategies. However, despite the many studies that have been completed, much remains to be learned about silica, its chemistry, and the cohesive forces that govern the structure types that it can adopt and their properties.

During the past decade, progress has been made in the mathematical modeling of silica with the calcula- tion of the structural and the elastic properties of several of its stable structure types. In these studies, the modeling of silica has been approached in two different but complimentary ways. On the one hand, relatively inexpensive calculations have been completed with empirical, semiempirical, and $a b$ initio based potential energy functions designed to mimic the force field of silica. For the most part, these calculations have been relatively successful despite their low computer costs in reproducing the structural and physical properties of several structure types of silica. ${ }^{3}$ On the other hand, periodic electronic structure calculations based on modified electron gas strategies, Hartree-Fock theory, and densityfunctional theory have also been completed. These calculations, albeit computationally much more expensive, have been successful in a number of cases in generating accurate static and dynamic properties for the silica structure types. ${ }^{4}$ However, one of the major drawbacks of both types of calculations is that they have been completed with a knowledge of the topologies of the structures to be modeled. No electronic structure calculations have been completed without a knowledge of the atomic coordinates of the structure. However, as observed above, the goal of computational modeling is not only to predict the properties of a given structure type, but also to derive the coordinates of the structure starting with a random arrangement of atoms. 
The atoms in a material tend to arrange themselves in order to minimize the energy of the resulting structure. In order to generate additional structure types, it seems a rather straightforward task to first generate a suitable energy functional and then to determine an efficient process to move the atoms about in such a way as to locate the minima on the Born-Oppenheimer potential energy surface that correspond to a low-energy structure. Boisen and co-workers used a combined simulated annealing and a quasi-Newton strategy to derive framework structure types for silica using a potential energy function based on the theoretical force field of the molecule $\mathrm{H}_{6} \mathrm{Si}_{2} \mathrm{O}_{7}{ }^{6} \mathrm{Each}$ calculation was initiated with four $\mathrm{SiO}_{2}$ formula units, randomly distributed in a unit cell. Using an algorithm that searches for global and local minimum-energy structures of a potential energy model for silica, more than 100 framework structure types were derived of which 23 were distinct. ${ }^{7}$ Despite the assumption of $P 1$ symmetry in the calculations, only 7 of the 23 local minimum-energy structures possess triclinic symmetry with those remaining exhibiting symmetries between $P c$ and $I \overline{4} 2 d$ to within $0.0001 \AA$. In these calculations, a structure type denoted $B G B 1$, was derived with $I \overline{4}$ space-group symmetry and a coordination sequence that is different from that of cristobalite and all of the other known silica structure types. It has a structure that consists of four- and eight-membered rings of silicate tetrahedra that are linked into an elegant framework by six-membered rings. As Boisen and co-workers found that the calculated energy for $B G B 1$, using the potential energy function matches that calculated for low-cristobalite, total-energy pseudopotential calculations were completed for the structure. In addition, calculations were completed for two other structure types ( $B G B 2$ and $B G B 3$ ), whose energies and coordination sequences match those calculated for low cristobalite. The $B G B 2$ structure has the true high cristobalite structure with space group $I \overline{4} 2 d$ and the $B G B 3$ structure is a stuffed derivative structure of cristobalite with space group $P n a 2_{1}$. This paper describes the results of the calculations and makes a comparison of the results with those obtained for low quartz, low cristobalite, silica sodalite, and stishovite.

\section{THE THEORETICAL METHODS OF MODELING}

The quantum mechanical modeling of silica is a computationally demanding task. The problems of deep pseudopotentials required to accurately model the oxide ion combined with the low symmetry and large unit cells of many of the structure types of silica made realistic and accurate quantum mechanical modeling of the structures intractable until recently. Also, because of the flat nature of the $\mathrm{SiO}$ bond length-SiOSi bond angle potential energy surface, the calculation of an accurate SiOSi bond angle for a silica structure type is very difficult. ${ }^{8}$

A significant advance that allowed the pseudopotential technique to be applied to much larger and complex systems than previously possible was made by Car and Parinello. ${ }^{9}$ They developed a quantum molecular dynamics technique that used an iterative approach to matrix diagonalization and showed how to derive an eigenvector that scales almost linearly instead of cubically with the number of plane waves used in a calculation. However, this technique still scales cubically with the number of atoms. This technique did not work for oxides, because of its use of local pseudopotentials. To circumvent this problem, Allan and Teter showed how oxides could be successfully modeled with a CPtype technique, using nonlocal pseudopotentials. ${ }^{10}$ Later, a preconditioned conjugate-gradient technique was developed for efficiently extracting eigenvectors that not only speeded up the calculation, but also allowed larger systems to be studied. ${ }^{11} \mathrm{~A}$ review of iterative diagonalization methods and the pseudopotential plane-wave method can be found in a recent review by Payne et $a .^{12}$ Recent significant advances in the application of the pseudopotential technique to silica and other oxides are the development of the extended-norm hardness-conserving (ENHC) (Ref. 15) and the Vanderbilt ultrasoft pseudopotential formalisms. The ENHC pseudopotential formalism has been shown to be considerably more accurate than norm conserving (NC) pseudopotentials when compared to the all-electron results. The Vanderbilt ultrasoft pseudopotentials offer the advantage of requiring significantly fewer plane waves than the ENHC and NC pseudopotentials, while still providing an impressive degree of accuracy as evident in a recent study of silica using this technique. ${ }^{26}$

This work was carried out using the BIOSYM PLANE_WAVE code. This code uses a preconditioned conjugate-gradient method to minimize the electronic degrees of freedom. The program solves for the electronic charge density using a density-functional framework ${ }^{13}$ within the local density approximation to electron exchange and correlation. The exchange-correlation term of the total energy is a rational polynomial fit to the Perdew-Wang fit, which itself reproduces the CeperleyAlder data. ${ }^{14}$ This form of the exchange-correlation function allows for continuous higher order derivatives, which makes it a more appropriate form for first-principles linear-response calculations. The electronic wave functions are expanded in a plane-wave basis set with periodic boundary conditions. Extended norm- and hardnessconserving (ENHC) pseudopotentials constructed within the scheme developed by Teter ${ }^{15}$ are used. This scheme ensures that the total energies of the atom and pseudoatom match to second order with respect to arbitrary changes in valence state occupancy. These potentials implement nonlocality with a separable form using double-reference states using Blöchl's generalization ${ }^{16}$ of the Kleinman and Bylander scheme ${ }^{17}$ and include scalar relativistic corrections using the technique proposed by Hamann. ${ }^{18}$ The oxygen potential was generated using neutral $2 s^{2} 2 p^{4}$ as the reference state with a radial cutoff of $1 \mathrm{bohr}$ for both the $s$ and $p$ components of the potential. The $s$ angular momentum channel was treated as a nonlocal component with two radial projection functions. The $p$ angular momentum channel was treated as the local component of the potential. The radius of the 
nonlinear core correction was $0.90 \mathrm{bohr}$. The silicon potential was generated using $3 s^{2} 3 p^{2} 3 d^{0}$ as a reference state with a radial cutoff of $1.9 \mathrm{bohr}$ for the $s, p$, and $d$ components of the potential. The $s$ and $p$ angular momentum channels were treated as nonlocal components of the potential with two radial projection functions each. The $d$ channel was treated as the local component of the potential. The radius of the nonlinear core correction was taken to be 1.70 bohr.

We have calculated the cohesive energy for each structure as a function of volume $/ \mathrm{SiO}_{2}$ formula unit. At a given volume, the positions of the cations and the anions were determined by minimizing the Hellmann-Feynman forces ${ }^{19}$ on the ions. The unit cell edges were determined by making adjustments, until the Pulay-corrected stress tensor was zero. The total energies were corrected using the finite-basis set correction method developed by Francis and Payne. ${ }^{20}$ The structural parameters were considered to be fully relaxed when the forces on the ions were less that $0.005 \mathrm{eV} / \AA$ and all stress tensor components were less than $0.001 \mathrm{eV} / \AA^{3}$. Calculations of this type were completed for a variety of volumes for each structure type. The resulting energies were fit to an expression of the form

$$
E=a+b V^{-1 / 3}+c V^{-2 / 3}+d V^{-1},
$$

which was used to estimate the pressure, the bulk modulus $\left(B_{0}\right)$, and the pressure derivative of the bulk modulus $\left(B_{0}^{\prime}\right)$. This expression was found to fit the data better than the Birch-Murnaghan or Birch ${ }^{21}$ equation of state.

In order to estimate with some degree of confidence the very small-energy differences obtained between the structure types, a convergence level of $0.1 \mathrm{mHartrees}$ per $\mathrm{SiO}_{2}$ unit was required in the calculations. The number of special $k$ points ${ }^{22-24}$ for the Brillouin zone integration was increased until the total energies had converged to $0.1 \mathrm{mHartrees}$ per $\mathrm{SiO}_{2}$ unit. Calculations were also completed at various kinetic energy cutoffs. It was determined that a cutoff of 60 Hartrees was required for a satisfactory convergence of the structural parameters. Further calculations on the the relaxed structures at a kinetic energy cutoff of 75 and 100 Hartrees show that the energy differences between the structures has converged to less than 0.0001 Hartrees at a kinetic energy cutoff of 75 Hartrees.

It was also found that the energies of the various structures converge at slightly different rates with respect to increasing kinetic energy cutoff. It is, therefore, necessary to carefully monitor these convergence rates in order to determine that the energy differences among various structures has stabilized.

\section{RESULTS AND DISCUSSIONS}

\section{A. Structural parameters}

\section{Stishovite}

Stishovite adopts the rutile structure and can be described as a distorted hexagonal close-packed array of oxide ions, in which one-half of the available octahedral sites are filled by $\mathrm{Si}$ cations. The $\mathrm{SiO}_{6}$ octahedra share opposite edges and form chains that run along c that are cross linked to equivalent chains by three-coordinate oxide ions as shown in Fig. 1. Stishovite is one of the most incompressible silicates known to man. It has two $\mathrm{SiO}_{2}$ formula units per unit cell and possesses $P 4_{2} / \mathrm{mnm}$ space-group symmetry. Stishovite is of geophysical interest as a possible constituent phase in the mantle and of general interest as being the simplest structure having octahedrally coordinated oxygen.

In our calculations for stishovite, we chose a [444] Monkhorst-Pack grid that translates into six special $k$ points in the irreducible Brillouin zone (IBZ). In the generation of the optimized structural parameters, a kinetic energy cutoff of 60 Hartrees was used, which corresponds to about 7000 plane waves in the basis set. The resulting structural parameters given in Table I are in close agreement with the room temperature and pressure results of the high-pressure single-crystal x-ray diffraction study of Ross et al. ${ }^{25}$ as well as previous theoretical studies using the Vanderbilt ultrasoft pseudopotentials ${ }^{26}$ and the linear augmented plane-wave method. ${ }^{27}$

In addition, structural parameters were calculated for simulated pressures up to $10 \mathrm{GPa}$. The resulting molar volume as a function of pressure is presented in Fig. 2 , with the results of some previous experimental and theoretical studies. ${ }^{25-28,30}$ The calculated structural parameters and bulk modulus for stishovite agree extremely well with the experimental data and with the results of previous calculations. High-pressure structural determinations reveal that the structure is anisotropic in its compression, with the structure compressing perpendicular to chains along $\mathbf{c}$ by about twice that along the chains. This structural response to pressure translates into an

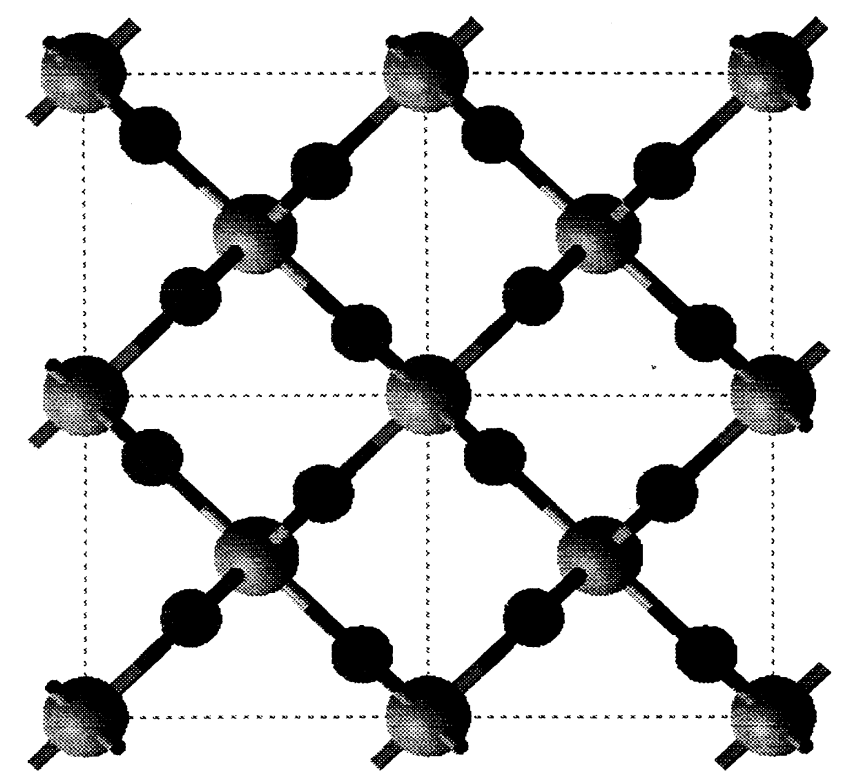

FIG. 1. Representation of the stishovite structure. Small and large spheres represent $\mathrm{O}$ and $\mathrm{Si}$ atoms, respectively. 
TABLE I. Structural parameters of stishovite.

\begin{tabular}{ccccc}
\hline \hline Parameter & Experiment & $\begin{array}{c}\text { This } \\
\text { work }\end{array}$ & $\begin{array}{c}\text { Previous } \\
\text { calculation }\end{array}$ & $\begin{array}{c}\text { Previous } \\
\text { calculation }\end{array}$ \\
\hline$a(\AA)$ & $4.1801^{\mathrm{a}}$ & 4.1804 & $4.1612^{\mathrm{b}}$ & $4.1626^{\mathrm{c}}$ \\
$c(\AA)$ & $2.6678^{\mathrm{a}}$ & 2.6651 & $2.6671^{\mathrm{b}}$ & $2.6641^{\mathrm{c}}$ \\
$\mathrm{O}(\mathrm{x})$ & $0.3067^{\mathrm{a}}$ & 0.3064 & $0.3055^{\mathrm{b}}$ & $0.3060^{\mathrm{c}}$ \\
$\mathrm{Si}-\mathrm{O}(1)(\AA)$ & $1.7564^{\mathrm{a}}$ & 1.7565 & $1.7573^{\mathrm{b}}$ & $1.7546^{\mathrm{c}}$ \\
$\mathrm{Si}-\mathrm{O}(2)(\AA)$ & $1.8130^{\mathrm{a}}$ & 1.8116 & $1.7979^{\mathrm{b}}$ & $1.8014^{\mathrm{c}}$ \\
$\mathrm{Si}-\mathrm{O}-\mathrm{Si}(\mathrm{deg})$. & $130.58^{\mathrm{a}}$ & 130.66 & $130.64^{\mathrm{b}}$ & $130.61^{\mathrm{c}}$ \\
$B_{0}(\mathrm{GPa})$ & $298^{\mathrm{d}}$ & 276 & $282^{\mathrm{b}}$ & $324^{\mathrm{c}}$ \\
$B_{0}^{\prime}$ & $4.0^{\mathrm{d}}$ & 6.8 & $5.6^{\mathrm{b}}$ & $4.0^{\mathrm{c}}$ \\
\hline \hline
\end{tabular}

${ }^{\text {a }}$ Reference 25 .

${ }^{\mathrm{b}}$ Reference 26.

${ }^{\mathrm{c}}$ Reference 27.

${ }^{\mathrm{d}}$ Reference 29.

increasing $c / a$ ratio with increasing pressure. Our theoretical calculations reproduce this result within the statistical error and agree with the experimental observation that the primary structural response to pressure is a decrease in the $\mathrm{Si}-\mathrm{O}$ bond lengths.

\section{Low quartz}

Low quartz is the stable phase of $\mathrm{SiO}_{2}$ at ambient conditions. Its structure as shown in Fig. 3, consists of trigonal helical chains of $\mathrm{SiO}_{4}$ tetrahedra that run parallel to $\mathrm{c}$ and are linked laterally into a tetrahedral framework structure. It has three $\mathrm{SiO}_{2}$ formula units per unit cell, and possesses $P 3_{2} 21$ space-group symmetry.

In our calculations, six special $k$ points were chosen at $\left(\frac{1}{6}, 0, \frac{1}{8}\right),\left(0, \frac{1}{3}, \frac{1}{8}\right),\left(\frac{1}{3}, \frac{1}{6}, \frac{1}{8}\right),\left(\frac{1}{3}, 0, \frac{3}{8}\right),\left(0, \frac{1}{6}, \frac{3}{8}\right),\left(\frac{1}{6}, \frac{1}{3}, \frac{3}{8}\right)$ with respective weights of $0.125,0.125,0.250,0.125,0.125$, and 0.250. A kinetic energy cutoff of 60 Hartrees corresponds to about 17000 plane waves in the basis set. The resulting structural parameters given in Table II agree well with the room temperature and pressure results of the high-pressure single-crystal x-ray diffraction study of Levien et al., ${ }^{31}$ the previous theoretical studies us-

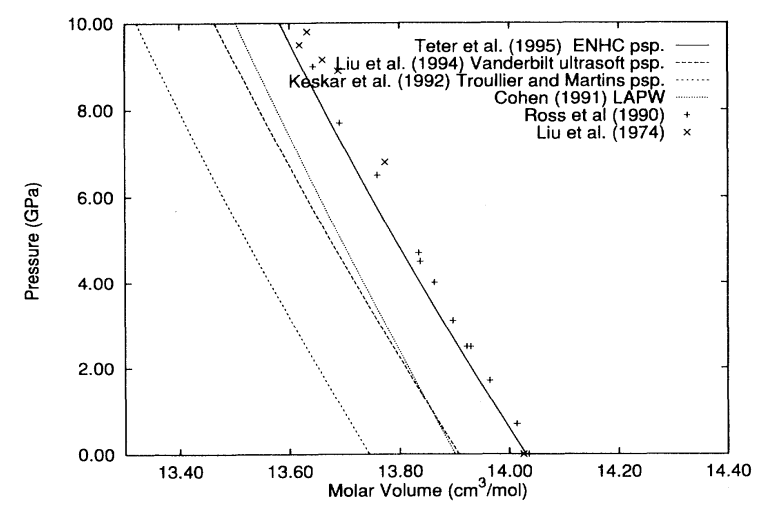

FIG. 2. Molar volume of the stishovite structure, as a function of pressure.

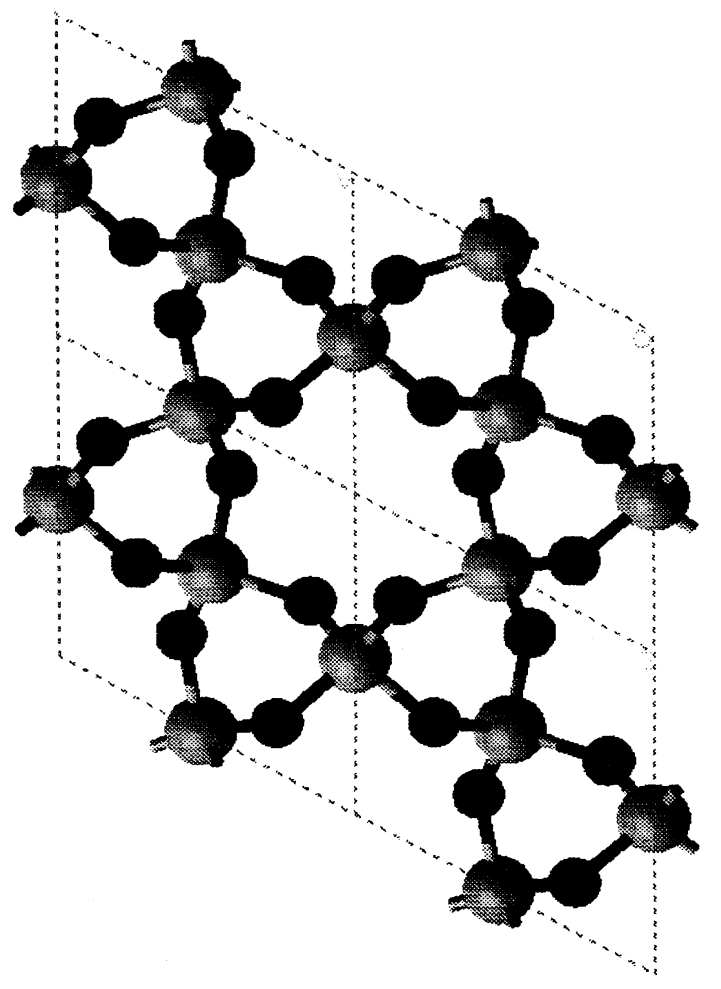

FIG. 3. Representation of the low-quartz structure. Small and large spheres represent $\mathrm{O}$ and $\mathrm{Si}$ atoms, respectively.

ing the Vanderbilt ultrasoft pseudopotentials, ${ }^{26}$ and the Troullier-Martins soft pseudopotentials. ${ }^{30}$

In addition, structural parameters were calculated for simulated pressures up to $15 \mathrm{GPa}$. The resulting molar volume as a function of pressure is plotted in Fig. 4, together with the results of some previous experimental and theoretical studies. ${ }^{26,30,32-35}$ The calculated struc-

TABLE II. Structural parameters of low quartz.

\begin{tabular}{ccccc}
\hline Parameter & Experiment & $\begin{array}{c}\text { This } \\
\text { work }\end{array}$ & $\begin{array}{c}\text { Previous } \\
\text { calculation }\end{array}$ & $\begin{array}{c}\text { Previous } \\
\text { calculation }\end{array}$ \\
\hline$a(\AA)$ & $4.9160^{\mathrm{a}}$ & 4.9541 & $4.8756^{\mathrm{b}}$ & $4.8900^{\mathrm{c}}$ \\
$c(\AA)$ & $5.4054^{\mathrm{a}}$ & 5.4414 & $5.4052^{\mathrm{b}}$ & $5.4900^{\mathrm{c}}$ \\
$\mathrm{Si}(\mathrm{u})$ & $0.4697^{\mathrm{a}}$ & 0.4750 & $0.4654^{\mathrm{b}}$ & $0.4690^{\mathrm{c}}$ \\
$\mathrm{O}(\mathrm{x})$ & $0.4135^{\mathrm{a}}$ & 0.4151 & $0.4125^{\mathrm{b}}$ & $0.4180^{\mathrm{c}}$ \\
$\mathrm{O}(\mathrm{y})$ & $0.2669^{\mathrm{a}}$ & 0.2584 & $0.2745^{\mathrm{b}}$ & $0.2740^{\mathrm{c}}$ \\
$\mathrm{O}(\mathrm{z})$ & $0.1466^{\mathrm{a}}$ & 0.1264 & $0.1143^{\mathrm{b}}$ & $0.1440^{\mathrm{c}}$ \\
$\mathrm{Si}-\mathrm{O}(1)(\AA)$ & $1.6046^{\mathrm{a}}$ & 1.6061 & $1.6077^{\mathrm{b}}$ & $1.6159^{\mathrm{c}}$ \\
$\mathrm{Si}-\mathrm{O}(2)(\AA)$ & $1.6137^{\mathrm{a}}$ & 1.6092 & $1.6153^{\mathrm{b}}$ & $1.6087^{\mathrm{c}}$ \\
$\mathrm{Si}-\mathrm{O}-\mathrm{Si}(\mathrm{deg})$. & $143.7^{\mathrm{a}}$ & 146.3 & $141.782^{\mathrm{b}}$ & $143.8^{\mathrm{c}}$ \\
$B_{0}(\mathrm{GPa})$ & $37.1^{\mathrm{a}}$ & 37.0 & $37.0^{\mathrm{b}}$ & $38.1^{\mathrm{c}}$ \\
$B_{0}^{\prime}$ & $6.2^{\mathrm{a}}$ & 3.2 & $4.3^{\mathrm{b}}$ & $3.9^{\mathrm{c}}$ \\
\hline \hline
\end{tabular}

${ }^{\mathrm{a}}$ Reference 31 .

${ }^{\mathrm{b}}$ Reference 26 .

${ }^{\mathrm{c}}$ Reference 30 . 


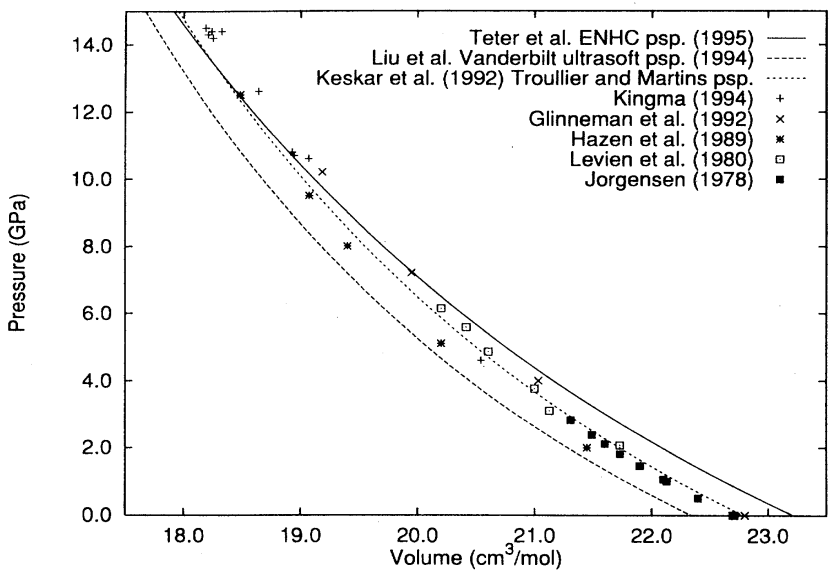

FIG. 4. Molar volume of the low-quartz structure, as a function of pressure.

tural parameters and bulk modulus agree well with experimental data and with the results of previous calculations. Our calculations reproduce the increasing $c / a$ ratio that is observed experimentally, they also agree with experimental observation that the primary structural response to pressure is a narrowing of the $\mathrm{Si}-\mathrm{O}-\mathrm{Si}$ bond angle and that the tetrahedra become more irregular with increasing pressure.

\section{Low cristobalite}

Low cristobalite is a relatively open tetrahedrally coordinated framework structure with $P 4_{1} 2_{1} 2$ space-group symmetry and four $\mathrm{SiO}_{2}$ formula units in the unit cell. A representation of the structure is shown in Fig. 5.

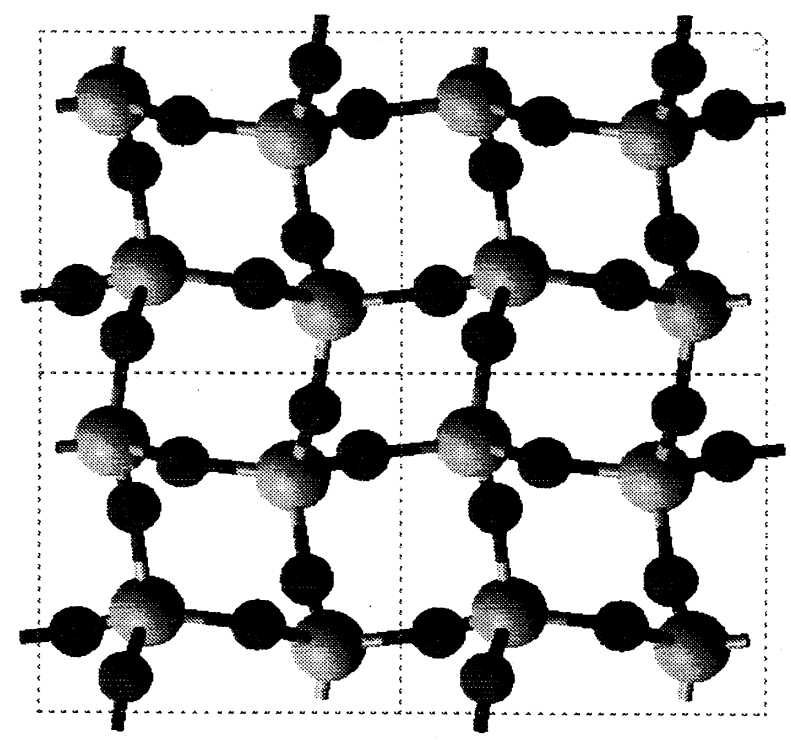

FIG. 5. Representation of the low-cristobalite structure. Small and large spheres represent $\mathrm{O}$ and $\mathrm{Si}$ atoms, respectively.

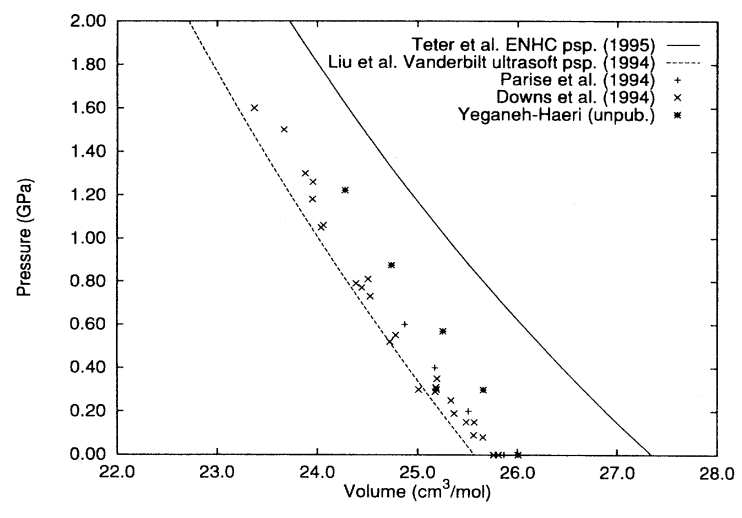

FIG. 6. Molar volume of the low-cristobalite structure, as a function of pressure.

In our calculations, a [444] Monkhorst-Pack grid was chosen, which translates into six special $k$ points in the IBZ. In the generation of the optimized structural parameters, a kinetic energy cutoff of 60 Hartrees was used, which corresponds to approximately 27000 plane waves in the basis set.

The structural parameters calculated for low cristobalite, given in Table III, compare moderately well with those measured at room temperature and pressure ${ }^{36}$ and those obtained in a theoretical study using the Vanderbilt ultrasoft pseudopotentials, ${ }^{26}$ but not as well as expected. The calculated bond lengths are realistic, but the calculated $\mathrm{Si}-\mathrm{O}-\mathrm{Si}$ bond angle is $\sim 6^{\circ}$ wider than the experimental value of $146.5^{\circ},{ }^{36}$ which translates into a larger unit cell than observed experimentally.

In addition, structural parameters were calculated for simulated pressures up to $2 \mathrm{GPa}$. The resulting molar volume, as a function of pressure, is presented in Fig. 6 with the results of some previous experimental and theoretical studies. ${ }^{26,30,36-38}$ The calculated bulk modulus of $11.9 \mathrm{GPa}$ agrees well with the experimental results. ${ }^{36}$

TABLE III. Structural parameters of low cristobalite.

\begin{tabular}{cccc}
\hline \hline Parameter & Experiment & $\begin{array}{c}\text { This } \\
\text { work }\end{array}$ & $\begin{array}{c}\text { Previous } \\
\text { calculation }\end{array}$ \\
\hline$a(\AA)$ & $4.9717^{\mathrm{a}}$ & 5.0630 & $4.9586^{\mathrm{b}}$ \\
$c(\AA)$ & $6.9222^{\mathrm{a}}$ & 7.0823 & $6.9074^{\mathrm{b}}$ \\
$\mathrm{Si}(u)$ & $0.3003^{\mathrm{a}}$ & 0.2895 & $0.3028^{\mathrm{b}}$ \\
$\mathrm{O}(x)$ & $0.2392^{\mathrm{a}}$ & 0.2431 & $0.2383^{\mathrm{b}}$ \\
$\mathrm{O}(y)$ & $0.1044^{\mathrm{a}}$ & 0.0833 & $0.1093^{\mathrm{b}}$ \\
$\mathrm{O}(z)$ & $0.1787^{\mathrm{a}}$ & 0.1687 & $0.1816^{\mathrm{b}}$ \\
$\mathrm{Si}-\mathrm{O}(1)(\AA)$ & $1.6034^{\mathrm{a}}$ & 1.6037 & $1.6113^{\mathrm{b}}$ \\
$\mathrm{Si}-\mathrm{O}(2)(\AA)$ & $1.6026^{\mathrm{a}}$ & 1.6037 & $1.6046^{\mathrm{b}}$ \\
$\mathrm{Si}-\mathrm{O}-\mathrm{Si}(\mathrm{deg})$. & $146.5^{\mathrm{a}}$ & 152.2 & $144.9^{\mathrm{b}}$ \\
$B_{0}(\mathrm{GPa})$ & $11.5^{\mathrm{a}}$ & 11.9 & $14.8^{\mathrm{b}}$ \\
$B_{0}^{\prime}$ & $9^{\mathrm{a}}$ & 3.0 & $2.4^{\mathrm{b}}$ \\
\hline \hline
\end{tabular}

${ }^{a}$ Reference 36.

${ }^{\mathrm{b}}$ Reference 26 . 
Our calculations reproduce the decreasing $c / a$ ratio that is observed experimentally and also agree with experiment in that the primary structural response to pressure is a narrowing of the $\mathrm{Si}-\mathrm{O}-\mathrm{Si}$ angle.

\section{Silica sodalite}

The sodalite structure type of silica is a very open tetrahedrally coordinated framework structure, which is composed of four- and six-member rings linked into cages that are packed together in a cubic close-packed array, and possesses $I m 3 m$ space-group symmetry with $12 \mathrm{SiO}_{2}$ formula units in the unit cell. It is an elegant structure consisting a framework of rigid cubo-octahedra, as shown in Fig. 7.

In our calculations for silica sodalite, a [222] Monkhorst-Pack grid was chosen that translates into one special $k$ point in the IBZ at $\left(\frac{1}{4}, \frac{1}{4}, \frac{1}{4}\right)$. In the generation of the optimized structural parameters, a kinetic energy cutoff of 60 Hartrees was used, which corresponds to approximately 108000 plane waves in the basis set. We were unable to calculate the cohesive energies at higher kinetic energy cutoffs, due to machine limitations.

Our calculated structural parameters for silica sodalite, presented in Table IV, compare reasonably well with the the room temperature and pressure results of the singlecrystal x-ray diffraction study of Richardson et al. ${ }^{39}$ In addition, structural parameters were calculated for simulated pressures up to $15 \mathrm{GPa}$. In order to calculate the total energies at various volumes, we chose to use a kinetic energy cutoff of 35 Hartrees. While this is not a suitable kinetic energy cutoff to use when comparing the

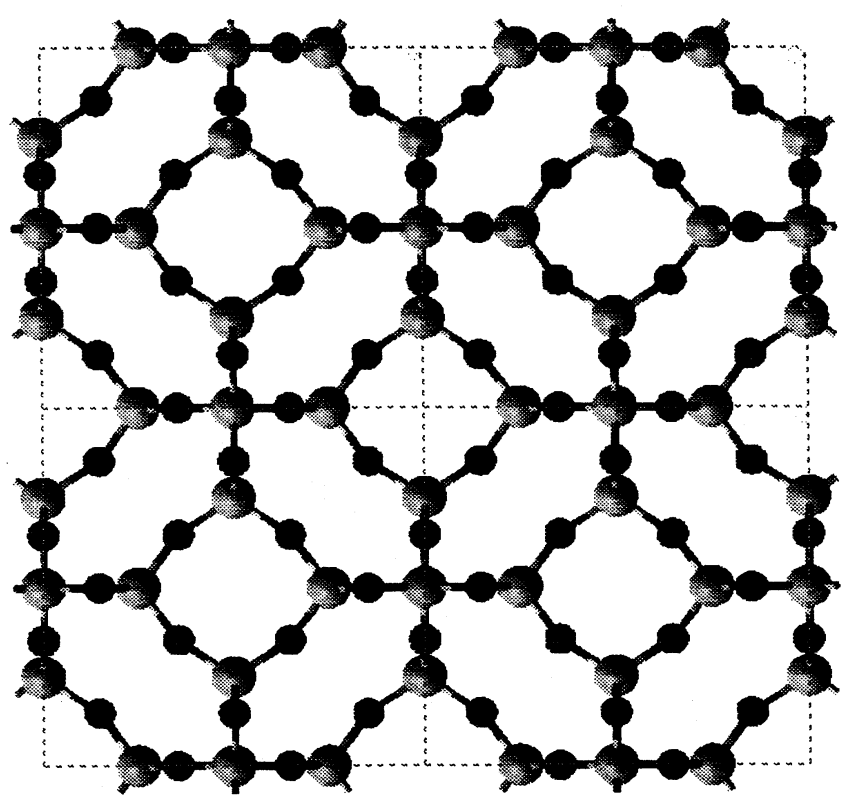

FIG. 7. Representation of the silica sodalite structure. Small and large spheres represent $\mathrm{O}$ and $\mathrm{Si}$ atoms, respectively.
TABLE IV. Structural parameters of silica sodalite.

\begin{tabular}{ccc}
\hline \hline & Experiment & $\begin{array}{c}\text { This } \\
\text { work }\end{array}$ \\
\hline$a(\AA)$ & $8.8273^{\mathrm{a}}$ & 8.9431 \\
$\mathrm{O}(y)$ & $0.6475^{\mathrm{a}}$ & 0.6477 \\
$\mathrm{Si}-\mathrm{O}(1)(\AA)$ & $1.5855^{\mathrm{a}}$ & 1.6068 \\
$\mathrm{Si}-\mathrm{O}-\mathrm{Si}(\mathrm{deg})$. & $159.6^{\mathrm{a}}$ & 159.5 \\
$B_{0}(\mathrm{GPa})$ & $\mathrm{a}$ & 122 \\
$B_{0}^{\prime}$ & $\mathrm{a}$ & 4.0 \\
\hline \hline
\end{tabular}

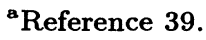

energy differences between structures, it is adequate for calculating the bulk modulus. We determined the bulk modulus of silica sodalite to be $122 \mathrm{GPa}$. This result conforms with the expected rigidity of the cubo-octahedral groups, the basic building block of the structure. The response of the silica-sodalite structure to pressure is a slight narrowing of the $\mathrm{Si}-\mathrm{O}-\mathrm{Si}$ bond angle accompanied by a relatively large decrease in the $\mathrm{Si}-\mathrm{O}$ bond length and an increase in the tetrahedral angle variance.

\section{Structure BGB1}

Structure $B G B 1$ is an elegant structure that consists of four- and eight-member rings that are linked into a framework by six-membered rings, as shown in Fig. 8. This structure has a more open structure than low cristobalite and a different coordination sequence than any of the known silica polymorphs. Structure $B G B 1$ has a body-centered tetragonal unit cell with $I \overline{4}$ symmetry and $8 \mathrm{SiO}_{2}$ formula units in the unit cell. The topology

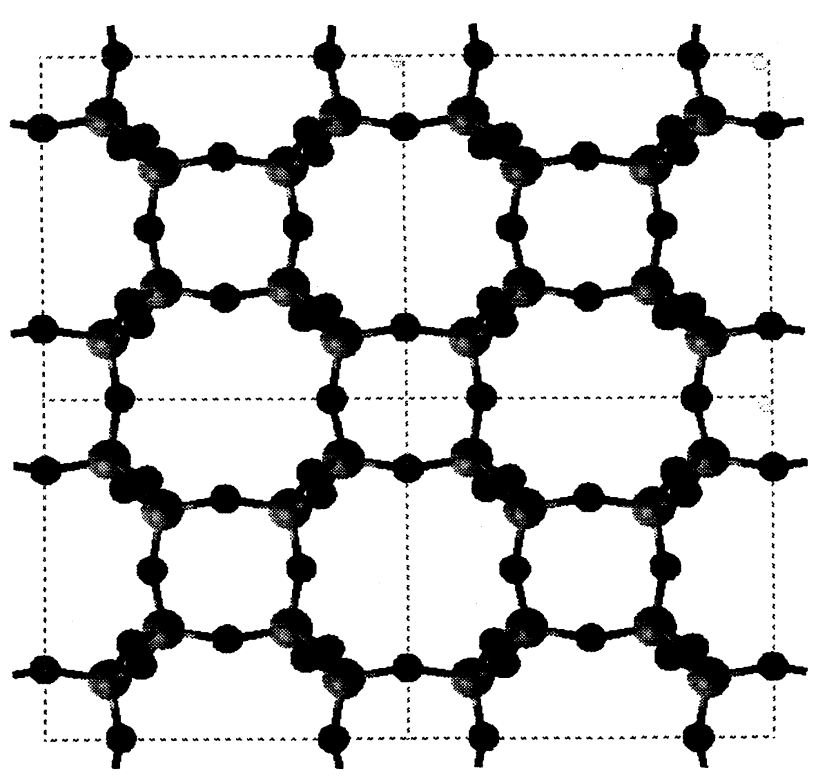

FIG. 8. Representation of the $B G B 1$ structure. Small and large spheres represent $\mathrm{O}$ and $\mathrm{Si}$ atoms, respectively. 
TABLE V. Structural parameters of $B G B 1$.

\begin{tabular}{cl}
\hline \hline & $\begin{array}{l}\text { This } \\
\text { work }\end{array}$ \\
\hline Parameter & 8.8153 \\
$a(\AA)$ & 5.0921 \\
$\mathrm{Si}(\boldsymbol{\AA}, y, z)$ & $0.1756,0.1770,0.0147$ \\
$\mathrm{O} 1(x, y, z)$ & $0.2632,0.2182,0.2808$ \\
$\mathrm{O} 2(x, y, z)$ & $0.7062,0.5022,0.4430$ \\
$\mathrm{Si}-\mathrm{O}(1)(\AA)$ & 1.6001 \\
$\mathrm{Si}-\mathrm{O}(2)(\AA)$ & 1.5999 \\
$\mathrm{Si}-\mathrm{O}(1)-\mathrm{Si}($ deg. $)$ & 157.3 \\
$\mathrm{Si}-\mathrm{O}(2)-\mathrm{Si}($ deg. $)$ & 151.8 \\
$B_{0}(\mathrm{GPa})$ & 21.5 \\
$B_{0}^{\prime}$ & 4.9 \\
\hline \hline
\end{tabular}

of the $B G B 1$ net is quite a common one. ${ }^{40}$ The $B G B 1$ structure has the same ring statistics and coordination sequence as the $\mathrm{B}$ net of $\mathrm{CrB}_{4},{ }^{41}$ the AlSi net of monoclinic $\mathrm{CaAl}_{2} \mathrm{Si}_{2} \mathrm{O}_{8},{ }^{42}$ and the net of all the atoms in $\beta$-BeO. ${ }^{43}$

In our calculations for $B G B 1$, we used the bodycentered unit cell and one special $k$ point at $\left(\frac{1}{4}, \frac{1}{4}, \frac{1}{4}\right)$. A convergence check using two special $k$ points shows that the structure is converged to the $0.1 \mathrm{mHartree}$ level with the one special $k$ point at $\left(\frac{1}{4}, \frac{1}{4}, \frac{1}{4} k\right.$. For the optimized structure presented, a kinetic energy cutoff of 60 Hartrees corresponds to approximately 60000 plane waves in the basis set.

The calculated structural parameters for $B G B 1$ are presented in Table V. In addition, structural parameters were calculated for simulated pressures up to $2 \mathrm{GPa}$. The $B G B 1$ structure is relatively soft, with the primary structural response to pressure being a significant decrease in the $\mathrm{Si}-\mathrm{O}-\mathrm{Si}$ bond angles from $157^{\circ}$ and $152^{\circ}$ to $139^{\circ}$, with the average $\mathrm{Si}-\mathrm{O}$ bond length remaining constant. The structure compresses anisotropically with the $c / a$ ratio decreasing with pressure.

\section{Structures BGB2 and BGB3}

The structure of $B G B 2$, shown in Fig. 9, is based on a body-centered tetragonal cell with $I \overline{4} 2 d$ symmetry and four $\mathrm{SiO}_{2}$ formula units in the unit cell. The structure of $B G B 3$, shown in Fig. 10, has an orthogonal unit cell with $P_{n a 2_{1}}$ symmetry and four $\mathrm{SiO}_{2}$ formula units in the unit cell. Structures $B G B 2$ and $B G B 3$ possess the cristobalite framework topology. ${ }^{5}$ Further supporting evidence that the structures have the cristobalite topology is provided by O'Keeffe and Hyde, ${ }^{43}$ who examined the space-group types that result by rotating the $\mathrm{SiO}_{4}$ tetrahedra about the $\overline{4}$ axes of the $C 9$ structure ( $F d 3 m$ space-group symmetry) originally proposed for high cristobalite. ${ }^{44}$ They described three structures with space-group types (I) $I \overline{4} 2 d$ (the true high-cristobalite structure), (II) $P 4_{1} 2_{1} 2$ or $P 4_{3} 2_{1} 2$ (the low-cristobalite structure), and (III) $P n a 2_{1}$ (a stuffed derivative structure referred to as the $P n a 2_{1}$ cristobalite type). Be-

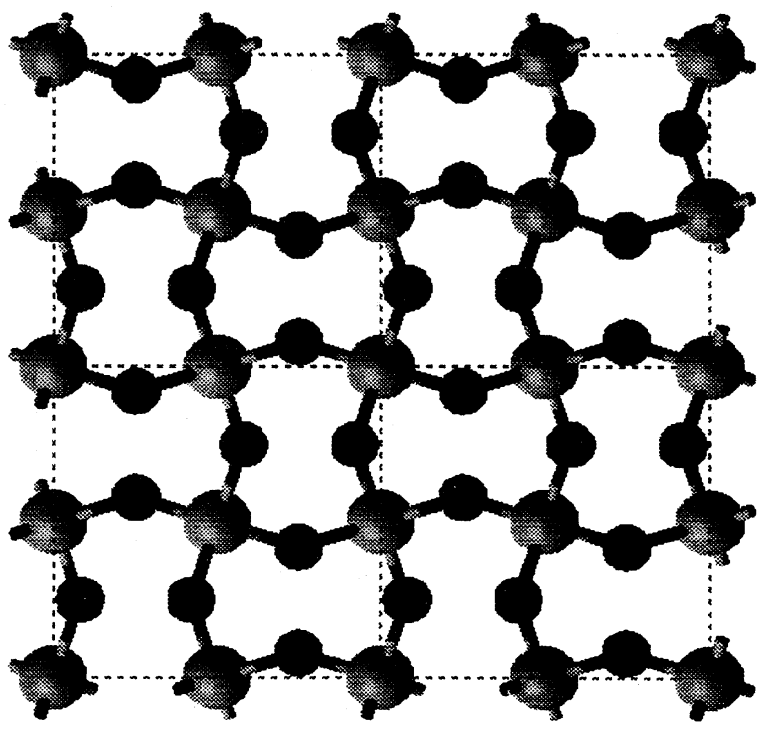

FIG. 9. Representation of the $B G B 2$ structure. Small and large spheres represent $\mathrm{O}$ and $\mathrm{Si}$ atoms, respectively.

cause the $B G B 2$ structure has a coordination sequence, a space-group type and a calculated powder diffraction pattern that match those of the true high-cristobalite structure, ${ }^{45}$ we consider these structures to be the same. Liu et al. ${ }^{46}$ presented the results of total-energy calculations on the $I 42 d$ (and others) structure of cristobalite

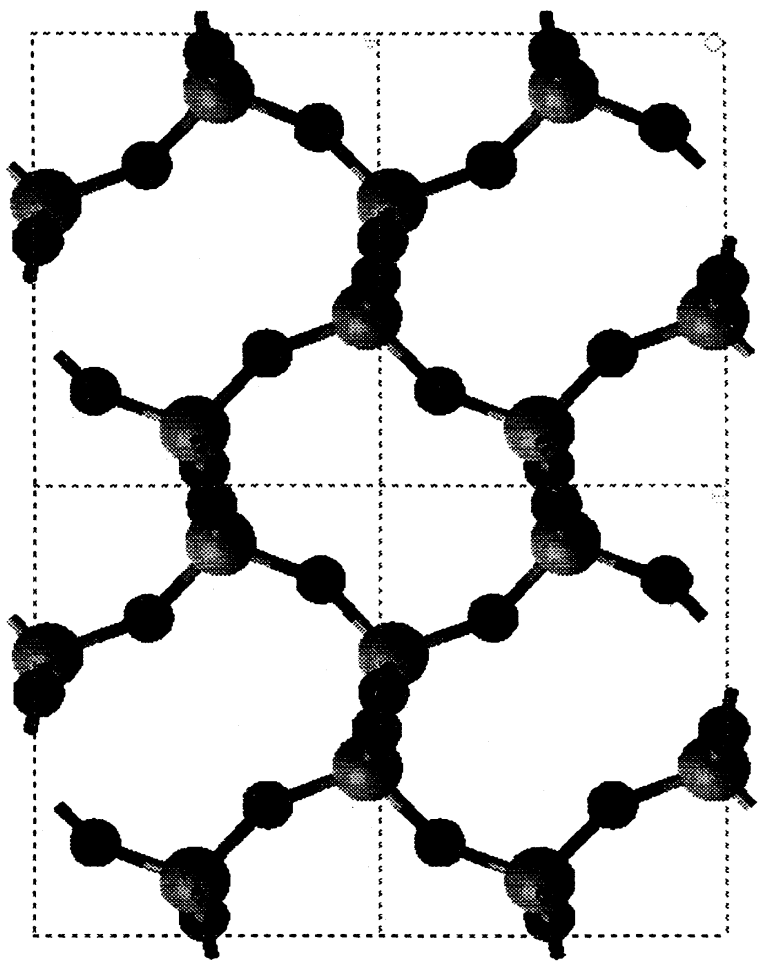

FIG. 10. Representation of the $B G B 3$ structure. Small and large spheres represent $\mathrm{O}$ and $\mathrm{Si}$ atoms, respectively. 
TABLE VI. Structural parameters of $B G B 2$.

\begin{tabular}{cl}
\hline & $\begin{array}{l}\text { This } \\
\text { work }\end{array}$ \\
Parameter & 5.0280 \\
$a(\AA)$ & 7.3033 \\
$c(\AA)$ & $0.5000,0.5000,0.5000$ \\
$\mathrm{Si}(x, y, z)$ & $0.2500,0.5798,0.3750$ \\
$\mathrm{O}(x, y, z)$ & 1.6045 \\
$\mathrm{Si}-\mathrm{O}(\AA)$ & 151.0 \\
$\mathrm{Si}-\mathrm{O}-\mathrm{Si}(\mathrm{deg})$. & 22.6 \\
$B_{0}(\mathrm{GPa})$ & 3.0 \\
$B_{0}^{\prime}$ & \\
\hline \hline
\end{tabular}

and determined the $I 42 d$ structure to be energetically feasible. As the $B G B 3$ structure has the same coordination sequence as cristobalite and the space group ( $\left.P n a 2_{1}\right)$ of the stuffed derivative structure, we consider these two structures to be the same. As silica with the cell dimensions, space group, and structure of $P n a 2_{1}$ cristobalite has yet to be reported, we could not compare our parameters with experimental results. However, the topology of tetrahedra and space-group symmetry is adopted by a number of stuffed derivative structures, such as $\mathrm{BeSiN}_{2}$ and $\mathrm{MgGeN}_{2} \cdot{ }^{43}$

For the $B G B 2$ and $B G B 3$ structures, we chose a [444] Monkhorst-Pack grid that translates into six special $k$ points in the IBZ for $B G B 2$ and eight special $k$ points in the IBZ for $B G B 3$. For the structurally relaxed $B G B 2$ and $B G B 3$ structures, this corresponds to approximately 27000 plane waves in the basis set.

The relaxed structural parameters for the $B G B 2$ and $B G B 3$ structures are presented in Tables VI and VII. Both of these structures have reasonable SiOSi bond angles and $\mathrm{SiO}$ bond lengths. The structural parameters for the $B G B 2$ structure were determined for simulated pressures of up to $5 \mathrm{GPa}$. The $B G B 2$ structure has a calculated bulk modulus of $22.6 \mathrm{GPa}$, which is between that of low quartz and low cristobalite. The primary response of the $B G B 2$ structure to pressure is a narrowing of the $\mathrm{Si}-\mathrm{O}-\mathrm{Si}$ bond angle with the $\mathrm{Si}-\mathrm{O}$ bond length remaining

TABLE VII. Structural parameters of $B G B 3$.

\begin{tabular}{cl}
\hline \hline Parameter & $\begin{array}{l}\text { This } \\
\text { work }\end{array}$ \\
\hline$a(\AA)$ & 5.1138 \\
$b(\AA)$ & 7.0562 \\
$c(\AA)$ & 5.0852 \\
$\mathrm{Si}(x, y, z)$ & $0.0373,0.6254,0.9997$ \\
O1 $(x, y, z)$ & $0.0105,0.5418,0.2918$ \\
O2 $(x, y, z)$ & $0.1733,0.2083,0.4585$ \\
$\mathrm{Si} 1-O 1(\AA)$ & 1.6042 \\
$\mathrm{Si} 2-\mathrm{O} 1(\AA)$ & 1.6030 \\
$\mathrm{Si3}-\mathrm{O} 2(\AA)$ & 1.6051 \\
$\mathrm{Si} 2-\mathrm{O} 2(\AA)$ & 1.6064 \\
$\mathrm{Si} 1-\mathrm{O} 1-\mathrm{Si} 2(\mathrm{deg})$. & 153.4 \\
$\mathrm{Si2}-\mathrm{O} 1-\mathrm{Si} 3($ deg.) & 150.1 \\
$B_{0}(\mathrm{GPa})$ & \\
$B_{0}^{\prime}$ & \\
\hline
\end{tabular}

constant. We were unable to determine the bulk modulus of the $B G B 3$ structure or its structural response to pressure, as the required calculations were intractable, due to the low symmetry of the structure.

\section{B. Energetics of structures}

In Fig. 11, we show the cohesive energies of stishovite, low quartz, low cristobalite, silica sodalite, and the theoretical structures $B G B 1, B G B 2$, and $B G B 3$ plotted as a function of volume at a kinetic energy cutoff of 60 Hartrees. The cohesive energies of the relaxed structures with increasing kinetic energy cutoff are shown in Table VIII. It can be seen that the differences in cohesive energy between structures have converged to below 0.1 mHartree at a kinetic energy cutoff of 75 Hartrees.

The relative differences in energies show that the $B G B 2$ phase is the most stable phase predicted solely by the cohesive energy at $0 \mathrm{~K}$. It is quite interesting to note that the internal energies for all of the relaxed tetrahedral structures examined are equivalent. The only structure examined that is significantly energetically different than the others is stishovite. The result that the stishovite structure has the highest cohesive energy agrees well with experimental enthalpy values determined by calorimetry. ${ }^{47}$ The result that low-quartz is not the lowest-energy tetrahedrally coordinated framework structure is unexpected as is the result that all the tetrahedral structures studied are energetically degenerate.

The small magnitude of the differences in these results forces one to consider the effect of the vibrational entropy contribution to the free energy when making comparisons to experimental data. Recent work using pair potentials ${ }^{49}$ and $a b$ initio linear-response theory ${ }^{48}$ to calculate the thermodynamic properties of silica polymorphs is important when trying to correlate the energetics of structures at $0 \mathrm{~K}$ with experimental results.

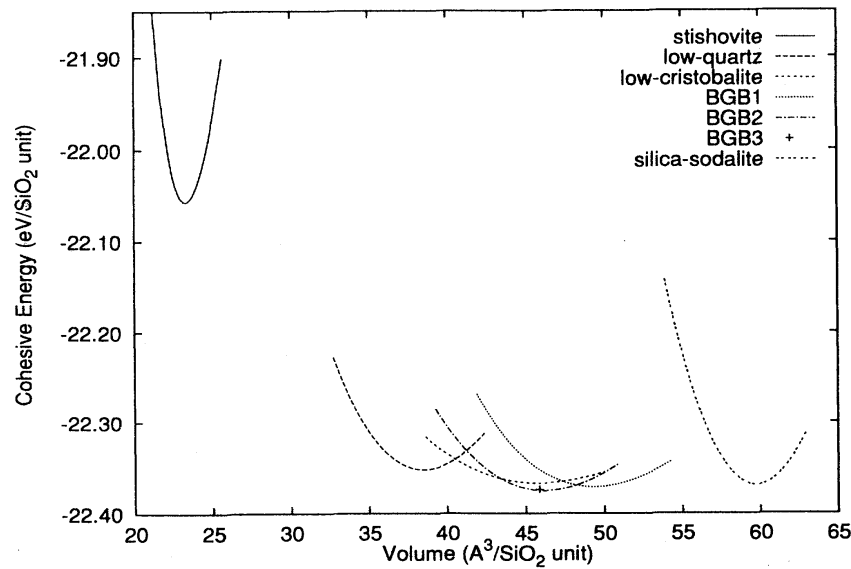

FIG. 11. Cohesive energies, as a function of volume for the structures studied. The curves were generated from fits to calculated data points using Eq. (1). 
TABLE VIII. Cohesive energies $\left(\mathrm{eV} / \mathrm{SiO}_{2}\right.$ unit) at various kinetic energy cutoffs.

\begin{tabular}{cccc}
\hline \hline & Cohesive energy & & \\
Structure & 60 Hartrees & 75 Hartrees & 100 Hartrees \\
\hline Stishovite & -22.056 & -22.098 & -22.110 \\
Low quartz & -22.352 & -22.396 & -22.409 \\
Silica sodalite & -22.363 & & \\
Low cristobalite & -22.366 & -22.412 & -22.426 \\
$B G B 1$ & -22.370 & -22.416 & -22.429 \\
$B G B 2$ & -22.374 & -22.420 & -22.433 \\
$B G B 3$ & -22.370 & -22.415 & -22.428 \\
\hline \hline
\end{tabular}

The recent work of Lee and Gonze using $a b$ initio linearresponse theory found that the contribution of zero-point energies to the difference in cohesive energy between stishovite and low quartz was $0.015 \mathrm{eV}$ per $\mathrm{SiO}_{2}$ unit. The effect of including temperature-dependent phonon contributions to the energetics of the tetrahedral structures is likely to be significant.

\section{CONCLUSIONS}

We have compared the structural properties and energetics of several hypothetical silica structures with stishovite, low quartz, low cristobalite, and silica sodalite, using state of the art first-principles total-energy calculations with highly accurate pseudopotentials and rigorous convergence criteria. We have shown that we are able to reproduce the structural properties of several well-characterized silica polymorphs and are, therefore, able to extend our calculations to determine the properties of several hypothetical silica structures with a reasonable degree of confidence.

Our calculations have shown that the cohesive energies for the hypothetical silica structures examined are equivalent to the cohesive energies of low quartz, low cristobalite, and silica sodalite. These results raise the possibility that there are a very large number of possible low-energy silica structures. The fact that only a relatively small number of these silica structures have been experimentally identified and characterized suggests that their existence is determined more from the direction of kinetics during formation than from the energetics of the final crystal structure. It is also quite possible that there remain a large number of silica structures yet to be discovered.

\section{ACKNOWLEDGMENTS}

The authors acknowledge the use of IBM RS/6000 workstations at the LASSP, the Cornell Materials Science Computing Center, and the Laboratory for Scientific Visualization at Virginia Tech. Computational results were obtained using the PLANE_WAVE program from BIOSYM Technologies. The National Science Foundation is thanked for partially supporting this work through Grant No. EAR-9303589.
1 J. Maddox, Nature 335, 201 (1988).

${ }^{2}$ M.L. Cohen, Nature 338, 291 (1989).

${ }^{3}$ C.R.A. Catlow and G.D. Price, Nature 347, 243 (1990).

${ }^{4}$ R.E. Cohen, Silica: Physical Behavior, Geochemistry and Materials Applications (Mineralogical Society of America, Washington, D.C., 1994), Vol. 29, p. 369.

${ }^{5}$ M.W. Deem and J.M. Newsam, J. Am. Chem. Soc. 114, 7189 (1992).

${ }^{6}$ M.B. Boisen and G.V. Gibbs, Phys. Chem. Min. 20, 123 (1993).

${ }^{7}$ M.B. Boisen, G.V. Gibbs, and M.S.T. Bukowinski, Phys. Chem. Min. 21, 269 (1994).

${ }^{8}$ G.V. Gibbs, E.P. Meagher, M.D. Newton, and D.K. Swanson, in Structure and Bonding in Crystals, edited by M. O'Keeffe and A. Navrotsky (Academic Press, New York, 1981), Vol. 1, p. 195.

${ }^{9}$ R. Car and M. Parinello, Phys. Rev. Lett. 55, 2656 (1985).

${ }^{10}$ D.C. Allan and M.P. Teter, Phys. Rev. Lett. 59, 1136 (1987)

${ }^{11}$ M.P. Teter, M.C. Payne, and D.C. Allan, Phys. Rev. B 40, 12255 (1989).

${ }^{12}$ M.C. Payne, M.P. Teter, D.C. Allan, T.A. Arias, and J.D. Joannopoulos, Rev. Mod. Phys. 64, 1045 (1992).

${ }^{13}$ W. Kohn and L.J. Sham, Phys. Rev. 140, 1133A (1965).

${ }^{14}$ D.M. Ceperly and B.J. Alder, Phys. Rev. Lett. 45, 566 (1980).
${ }^{15}$ M.P. Teter, Phys. Rev. B 48, 5031 (1993).

${ }^{16}$ P.E. Blöchl, Phys. Rev. B 41, 5414 (1990).

${ }^{17}$ L. Kleinman and D.M. Bylander, Phys. Rev. Lett. 48, 1425 (1982).

${ }^{18}$ D.R. Hamann, Phys. Rev. B 41, 2980 (1989).

${ }^{19}$ R.P. Feynman, Phys. Rev. 56, 340 (1939).

${ }^{20}$ G.P. Francis and M.C. Payne, J. Phys. Condens. Matter 17, 1643 (1990).

${ }^{21}$ F. Birch, J. Geophys. Res. 83, 1257 (1978).

22 D.J. Chadi and M.L. Cohen, Phys. Rev. B 8, 5747 (1973).

${ }^{23}$ H.J. Monkhorst and J.D. Pack, Phys. Rev. B 13, 5188 (1976).

${ }^{24}$ R.A. Evarestov and V.P. Smirnov, Phys. Status Solidi 119, 9 (1983).

${ }^{25}$ N.L. Ross, J.F. Shu, R.F. Hazen, and T. Gasparik, Am. Min. 75, 739 (1990).

${ }^{26}$ F. Liu, S.H. Garofalini, R.D. King-Smith, and D. Vanderbilt, Phys. Rev. B 49, 12528 (1994).

${ }^{27}$ R.E. Cohen, Am. Min. 76, 733 (1991).

${ }^{28}$ L. Liu, W.A. Bassett, and T. Takahashi, J. Geophys. Res. 79, 1160 (1974).

${ }^{29}$ J.F. Shu et al., Silica: Physical Behavior, Geochemistry and Materials Applications (Ref. 4), Vol. 29, p. 47.

${ }^{30}$ N.R. Keskar and J.R. Chelikowsky, Phys. Rev. B 46, 1 (1992).

${ }^{31}$ L. Levien, C.T. Prewitt, and D.J. Weidner, Am. Min. 65, 
920 (1980).

${ }^{32}$ K.J. Kingma, Ph.D. dissertation, Johns Hopkins University, Baltimore, MD, 1994.

33 J. Glinnemann, H.E. King, Jr., H. Schultz, T. Hahn, S.J. La Placa, and F. Dacol, Z. Kristallogr. 198, 177 (1992).

${ }^{34}$ R.M. Hazen, L.W. Finger, R.J. Hemley, and H.K. Mao, Solid State Commun. 72, 507 (1989).

35 J.D. Jorgensen, J. Appl. Phys. 49, 5473 (1978).

${ }^{36}$ R.T. Downs and D.C. Palmer, Am. Min. 79, 9 (1994).

37 J.B. Parise, A. Yeganeh-Haeri, D.J. Weidner, J.D. Jorgensen, and M.A. Saltzberg, J. Appl. Phys. 75, 1361 (1994).

38 A. Yeganeh-Haeri (private communication).

39 J.W. Richardson, Jr., J.J. Pluth, J.V. Smith, W.J. Dytrych, and D.M. Bibby, J. Phys. Chem. 92, 243 (1988).

${ }^{40} \mathrm{M}$. O'Keeffe (unpublished).
${ }^{41}$ S. Andersson and T. Lunström, Acta Chem. Scand. 22, 3103 (1968).

${ }^{42}$ Y. Takeuchi, N. Haga, and J. Ito, Z. Kristallogr. 137, 380 (1973).

${ }^{43}$ M. O'Keeffe and B.G. Hyde, Acta Crystallogr. Sec. B 32, 2923 (1976).

${ }^{44}$ R.W.G. Wyckoff, Am. J. Sci. 9, 448 (1925).

45 D.R. Peacor, Z. Kristallogr. 138, 274 (1973).

${ }^{46}$ F. Liu, S.H. Garofalini, R.D. King-Smith, and D. Vanderbilt, Phys. Rev. Lett. 70, 2750 (1993).

${ }^{47}$ I. Petrovic, A. Navrotsky, M.E. Davis, and S.I. Zones, Chem. Mater. 5, 1805 (1994).

${ }^{48}$ C. Lee and X. Gonze, Phys. Rev. B 51, 8610 (1995).

${ }^{49}$ N.R. Keskar and J.R. Chelikowsky, Phys. Chem. Min. 22, 223 (1995). 